\title{
STUDY OF THE FLIGHT OF SMALL LIQUID DROPLETS THROUGH A THIN LIQUID FILM FOR PICOLITRE LIQUID TRANSFER
}

\author{
Laurent Geyl ${ }^{l}$, Gustav Amberg ${ }^{2}$, Wouter van der Wijngaart ${ }^{l}$ and Göran Stemme ${ }^{l}$ \\ ${ }^{1}$ Microsystem Technology, School of Electrical Engineering, \\ ${ }^{2}$ School of Mechanical Engineering, \\ Royal Institute of Technology, Stockholm, SWEDEN; email: wouter@s3.kth.se
}

\begin{abstract}
We introduce and successfully demonstrate a novel method and system for subsequent dispensing, mixing and ejecting of picolitre liquid samples in a single step. The system consists of a free liquid film, suspended in a frame and positioned in front of a droplet dispenser. In this study we tested and modelled the flight of liquid droplets, ejected from an inkjet print head, through a suspended liquid film. Model and experiment are in accordance.
\end{abstract}

\section{INTRODUCTION}

Dispensing, mixing and ejecting of picolitre volume sample are operations which are of importance in applications such as high-throughput drug screening and the generation of microarrays.

The generation of ink droplets in the picolitre range forms a core technology in the field of publishing technology. The use of this technology for biotechnical applications is common, however, not uncomplicated due to incompatibility between the printhead operation (heat, electric field, pneumatic pressure...) and the liquid properties of biosensitive sample (viscosity, conductivity, saturation pressure...). Moreover, the dead volume of the dispenser may require more liquid sample for priming the device than is physically available or than is economically viable.

Previously, dispensing through liquid layers has been demonstrated [1] in which a liquid sheet was guided over the nozzle surface of a dispenser. In this approach, premature mixing of the liquids will occur through diffusion at the liquid-liquid interface. Moreover, the dispensing characteristics depend on the properties of both dispensed liquids. Another related technology is the TopSpot ${ }^{\circledR} 4$ [2], in which an air pressure pulse generates an array of droplets from open microfluidic ducts.

In this paper we introduce a novel method and system for ejecting picolitre amount of liquid from a very small initial sample volume. The sample volume is not in contact with other liquids prior to dispensing, thus there is no risk for sample dilution or contamination. Moreover, the technology has the potential to mix and dispense more than one liquid in a single dispensing step.

\section{DEVICE DESCRIPTION}

The novel system consists of a droplet dispenser and one or more free liquid film(s) suspended in a frame in front of the dispenser, much like a soap film in a soap bubble frame (Fig. 1). Droplets ejected by the dispenser fly through the liquid film(s). The ejected droplets and the film liquid mix partially while the droplets pass through, thus the initial "dispenser droplet" forms a transport medium for the liquid in the free liquid film. When the resulting "film droplet" is captured on a target plate, it can be used for further analysis.

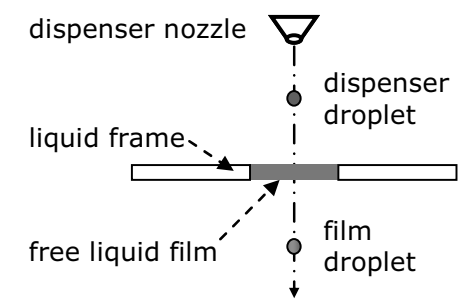

Fig. 1. Cross-sectional schematic of the new method for sample transport. A dispenser droplet flies through a freestanding liquid film, suspended in a frame, and transports part of the film liquid with it.

Forming a free liquid film on the macro scale typically requires low surface tension (soap) liquid. On the microscale, the liquid frame is relatively thick compared to the span of the free liquid film, making the formation of microscale free liquid films uncomplicated. The liquid in the dispenser can typically be a buffer solution that is available in larger quantities. The liquid volume required to form the free liquid film is typically much smaller than the volume required priming a dispenser. This method is thus suited for applications where the amount of sample available is limited. The minimum initial sample volume required equals the amount necessary to form the free liquid film.

Another benefit of the novel approach is that the buffer liquid in the dispenser can be tailored for reliable dispensing characteristics. For example, dispense liquids should not foul the internal dispenser surface and have appropriate surface tension characteristics. Only few dispense liquids are required to serve as transport medium for a multitude of sample liquids. Thus, this novel approach eases adapting available ink-jet printheads for dispensing of a large range of liquids of interest in biotechnology. 
The method forms a general approach to transport picolitre sample volume from a microfluidic (lab-on-chip) environment to another carrier, for example for further analysis. For this purpose, the microfluidic system must be modified with an interfacing window to allow the flight of dispenser droplets through the system, as illustrated in Fig. 2.

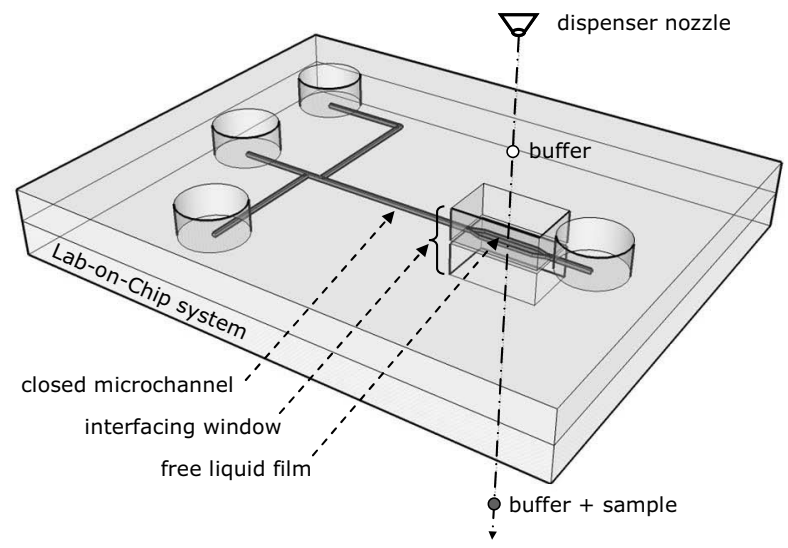

Fig. 2. Conceptual view of a lab-on-chip system adapted with an interfacing window to enable sample extraction using the novel method.

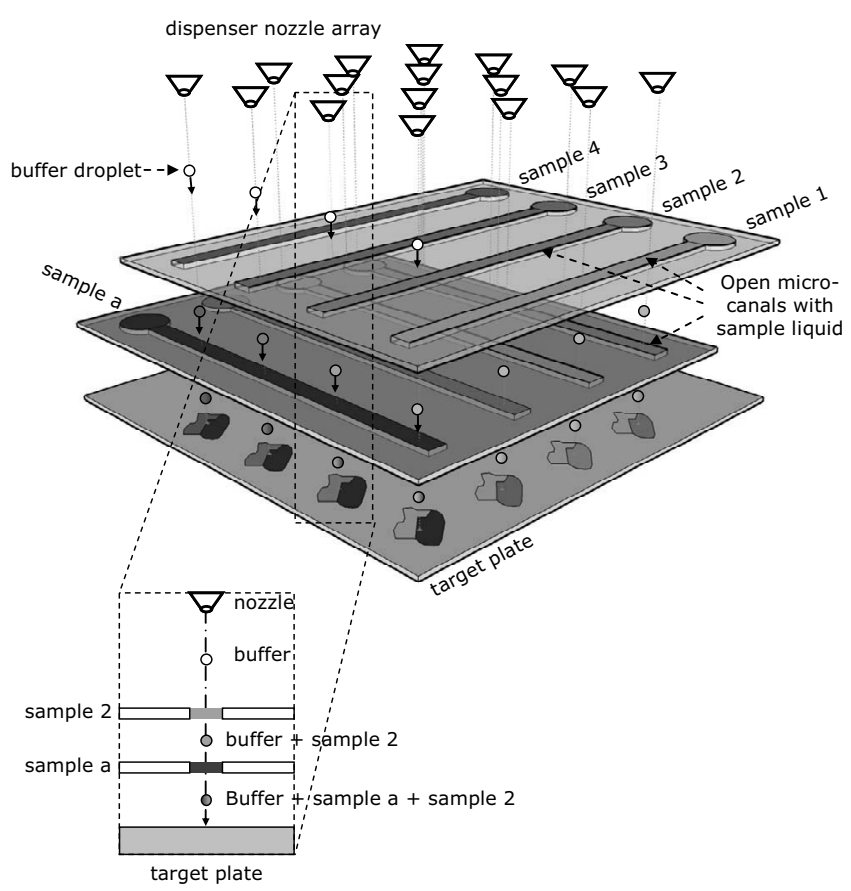

Fig. 3. Conceptual view on a system for generating microarrays of sample mixtures. Each nozzle in the array fires droplets of buffer liquid through two liquid films (open microcanals without "floor" and "ceiling") placed in the path of the droplet. Each liquid film contains sample liquid, which partially mixes with the passing buffer droplet. After passing the two liquid films, the droplet thus consists of a mixture of buffer and both samples. Capturing these droplets on a target plate forms an array of unique sample mixtures.

Parallelising this scheme renders a versatile system for producing microarrays, as illustrated in Fig. 3. In the illustrated system, the dispenser droplet flies through two free liquid films before being captured on a target plate.

\section{MEASUREMENT SETUP}

Our experimental work investigated the flight of small liquid droplets through a thin liquid film. For this purpose we adapted the standard printhead test equipment of the printhead manufacturer XAAR. The setup consists of a rectangular glass frame positioned in front of a commercial piezoelectric inkjet printhead (Xaar XJ128), as illustrated in Fig. 4. The glass frame formed a $1.25 * 1.75 \mathrm{~mm}^{2}$ square window for suspending a free liquid film. The measurement setup further incorporated a stroboscopic camera for visualisation and measurement of droplet velocity and volume. The piezoelectric XJ128 printhead can only handle non-conductive liquids with an appropriate surface tension. We therefore chose oily ink as liquid during this proof-ofconcept investigation. This had the additional benefit that the evaporation rate was limited, making the formation of stable liquid films easier. The printhead was filled with transparent ink; the free liquid film consisted of black ink. Both the black and the transparent ink have a viscosity $\mu=0.01 \mathrm{Ns} \cdot \mathrm{m}^{-2}$, a density $\rho=880 \mathrm{~kg} \cdot \mathrm{m}^{-3}$ and a surface tension $\gamma=0.032 \mathrm{~N} \cdot \mathrm{m}^{-1}$.

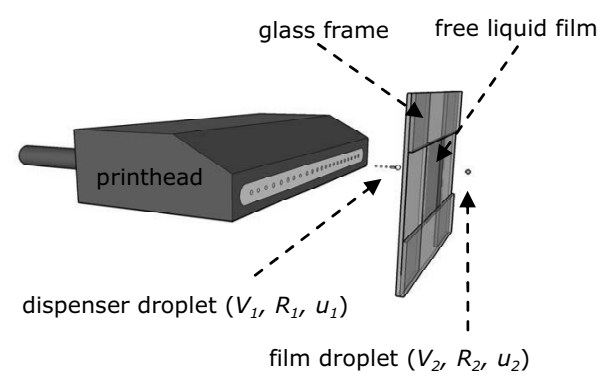

Fig. 4. Schematic of the experimental setup used to study the flight of a dispenser droplet through a suspended free liquid film.

An additional setup was built for measuring the thickness of the suspended black ink film. It consists of two needles, each mounted via a micropositioner on a common base plate (Fig. 5). The needles can be moved perpendicularly towards the liquid film and it was visually observed when the needles contacted the film. When both needle tips have just reached contact with the film, their inbetween distance equals the film thickness.

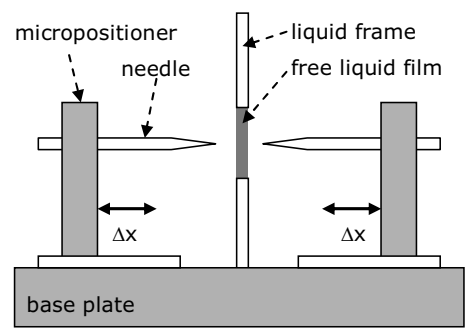

Fig. 5. Measurement setup to determine the thickness of the free liquid film. 


\section{EXPERIMENTAL}

First we measured the thickness of the black ink film suspended in the glass frame. In forty-nine independent measurements the liquid film thickness $h$ varied between 39 $\mu \mathrm{m}$ and $121 \mu \mathrm{m}$, with an average estimated thickness $h=75 \pm 24 \mu \mathrm{m}$.

Thereafter we studied the flight of dispenser droplets through a liquid film. For this purpose, we fired droplets at a fixed frequency of $50 \mathrm{~Hz}$. The velocity of the dispenser droplets was varied by varying the operation voltage. No measurable change in radius, and thus volume, of the dispenser droplets was observed with varying operation voltage. The dispenser droplet radius and volume were $R_{l} \approx 27 \mu \mathrm{m}$, and $V_{l} \approx 80 \mathrm{pL}$, respectively. The dispenser droplet velocity, $u_{l}$, was varied between 4 and $11 \mathrm{~m} \cdot \mathrm{s}^{-1}$. When the dispensing velocity, $u_{l}$, was below a critical velocity $u^{*}=6.6 \mathrm{~m} \cdot \mathrm{s}^{-1}$, the droplet merges with the free liquid film upon impact. When $u_{1}>u^{*}$, the droplet flies through the liquid film and a film droplet appears on the other side, as shown in Fig. 6.

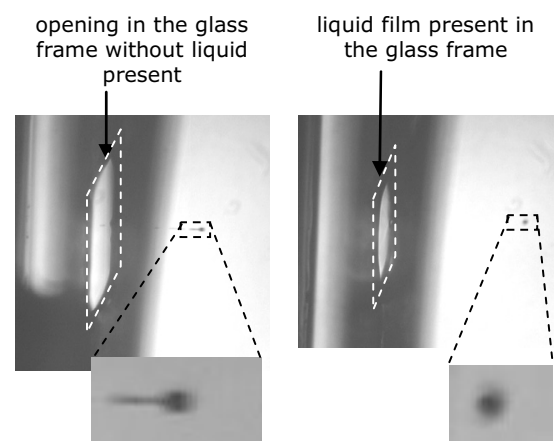

Fig. 6. Microscope pictures of a dispenser droplet with satellites, ejected prior to filling the frame with a liquid film (left), and a film droplet, i.e. after passing through a free liquid film, under the same dispensing conditions (right).

One can observe that the printhead creates satellites when operated at high voltages. However, after flying through the liquid film, no satellites remain. The system thus functions as a filter for satellite droplets. A change in the size, $R_{2}$, of the film droplet compared to the dispenser droplet was not significant $\left(R_{1} \approx R_{2}\right)$. However, the film droplet velocity, $u_{2}$, was strongly dependent on the dispensing velocity, $u_{1}$, as shown in Fig. 7.

Note that when the residual velocity $u_{2}>u^{*}$, it should theoretically be possible for the droplet to fly through a second free liquid film. This occurs for dispensing velocities $u_{l}>11 \mathrm{~m} \cdot \mathrm{s}^{-1}$, but has not yet been tested.

Furthermore, the successful dispensing of parallel droplets through the same liquid film was shown (Fig. 8).

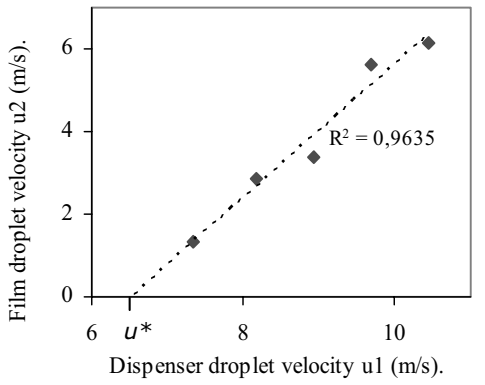

Fig. 7. The measured film droplet velocity $u_{2}$ plotted vs. the dispensing velocity $u_{1}$. Linear regression shows the critical velocity $u^{*} \approx 6.6 \mathrm{~m} \cdot \mathrm{s}^{-1}$ required to generate film droplets.

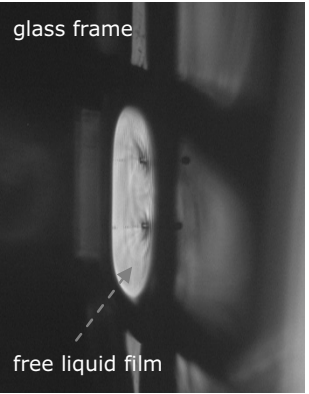

Fig. 8. Photograph showing two droplets passing the same film simultaneously.

In another experiment, the transport of liquid from the free film to the film droplet was measured using greyscale analysis of the film droplets. For this purpose, the film droplets were collected as printed dots on a paper substrate in periods of one second, i.e. every second, a new printed dot was formed containing 20 film droplets. During continuous operation of the system, the black ink in the free film becomes locally saturated with transparent dispensing ink at the point where the droplets fly through it. Therefore, the subsequently created dots contain less and less black ink, i.e. they become lighter in tone, as illustrated in Fig. 9.

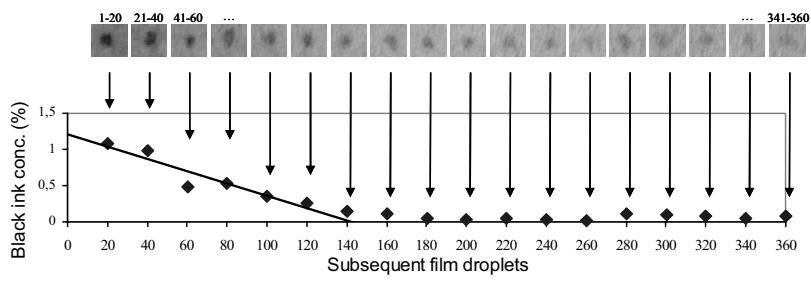

Fig. 9. Estimation through greyscale analysis of the liquid transfer from the film to the droplet. The 18 greyscale pictures (top) show ink dots on paper, each dot made by 20 consecutive film droplets. The bottom plot shows the respective black ink concentration.

Greyscale analysis allows to estimate the transport of liquid from the film to the eject droplet as $1.2 \%$. I.e., every $80 \mathrm{pL}$ film droplet contains approximately $1 \mathrm{pL}$ liquid from the free liquid film. 


\section{THEORETICAL MODELLING AND DISCUSSION}

Let's start by assuming that the interaction between the droplet and the film is limited during the passage, i.e. the droplet moves rather unhindered through the free liquid film. This assumption allows us to model the droplet as a solid sphere moving through a fluid, with a flow profile similar to that of steady flow around a spherical object. The Reynolds number for a velocity $u_{l} \approx 10 \mathrm{~m} \cdot \mathrm{s}^{-1}$ and droplet diameter $2 R_{l} \approx 54 \mu \mathrm{m}$ is $R e \approx 53$, i.e. the system is in the laminar regime.

The boundary layer thickness $\delta \propto \sqrt{\frac{\mu}{\rho} \cdot \frac{l}{u}}$ can be interpreted as the distance around a solid object in a flow field where the flow profile changes considerably from the profile that would occur if the object would not be present. $l$ is a characteristic length, typically the distance to the leading edge of the object. Using $R$ as the characteristic length $l$, and $u=u_{1}=10 \mathrm{~m} \cdot \mathrm{s}^{-1}$, gives us the estimate $\delta \approx 5.2 \mu \mathrm{m}$. The boundary layer thickness is also a measure for the region of the droplet and the film that will influence one another, for example through mixing. The fact that $\delta<R_{l}$ indicates that modelling the droplet as a solid sphere is not unreasonable.

The system can be described using the laws of energy conservation, taking into consideration the kinetic energy, $E_{k}=\frac{m \cdot u^{2}}{2}$, and the surface tension energy, $E_{s}=4 \pi R^{2} \gamma$, of both the dispensed droplet (index 1) and film droplet (index 2):

$$
E_{k, 1}+E_{s, 1}=E_{\text {loss }}+E_{k, 2}+E_{s, 2} \text {. }
$$

The term $E_{\text {loss }}$ includes the energy losses occurring when the droplet moves through the liquid film. The surface tension energy terms are equal $\left(R_{I} \approx R_{2}=R\right)$ and much smaller than the kinetic energy of the dispenser droplet, and they can thus be removed. Using the solid sphere model and neglecting acoustic and capillary wave losses, the total energy loss of the droplet can be contributed to viscous losses and can be expressed using Stokes' equation for drag:

$F_{d r a g}=-6 \pi \mu R u(x)=m \frac{d u(x)}{d t}$,

with $m=\frac{4}{3} \pi R^{3} \rho$ the droplet mass. The use of eq. 2 could be questionable here, since it is strictly valid only when the Reynolds number is less than unity. However, in the present Reynolds number range, $1<\operatorname{Re}<50$, eq. 2 still captures the dependency of fluid drag on velocity better than the corresponding high Reynolds number formula. In view of the many other uncertainties here, it was not deemed meaningful to use a more accurate (and complicated) model for the drag, even though such are certainly available. The droplet position and velocity $u(x)=\frac{d x}{d t}$ can thus be expressed as

$x(t)=\frac{m}{6 \pi \mu R} u_{1}\left[1-\exp \left(-\frac{6 \pi \mu R}{m} t\right)\right]$, and $u(t)=u_{1} \exp \left(-\frac{6 \pi \mu R}{m} t\right)=u_{1}-\frac{6 \pi \mu R}{m} x(t)$.

For a film droplet to appear after moving through the complete film thickness $h$, the condition

$\left.u\right|_{x=h}=u_{1}-\frac{6 \pi \mu R}{m} h>0$

must be fulfilled. Thus, the theoretical critical velocity $u^{*}=\frac{9}{2} \frac{\mu h}{\rho R^{2}}=5.26 \mathrm{~m} \cdot \mathrm{s}^{-1}$, which apparently is in accordance with the experimentally obtained value of $6.6 \mathrm{~m} \cdot \mathrm{s}^{-1} . E_{\text {loss }}$ can now be expressed as

$E_{\text {loss }}=6 \pi \mu R \int_{0}^{h} u(x) d x=6 \pi \mu R h \cdot\left[u_{1}-u^{*}\right]$

In Fig. 10, both the theoretical and the measured kinetic energy of the film droplet are plotted versus the dispensing velocity. A good match between measurement and theory can again be observed.

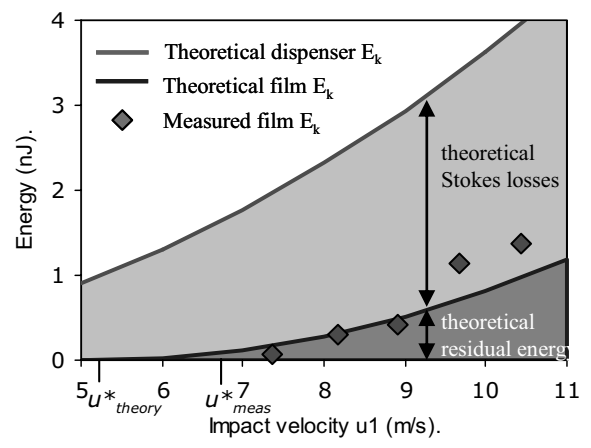

Fig. 10. The theoretical and measured kinetic energy of the dispenser droplets and the film droplets vs. the dispensing velocity.

\section{CONCLUSIONS}

We introduced and successfully demonstrated a novel method and system for subsequent dispensing, mixing and ejecting of picolitre liquid samples in a single step. We also presented a theoretical model of the flight of dispensed liquid droplets through a suspended liquid window that was in accordance to experimental results.

\section{ACKNOWLEDGEMENTS}

We would like to thank XAAR and Vinnova for their support through the Swedish Centre for Surface and Microstructure Technology, SUMMIT.

\section{REFERENCES}

[1] J. Nilsson et al., Proceedings $\mu$ TAS 2001, Monterey, Canada, pp 75-77.

[2] TopSpot ${ }^{\circledR}$ technology is developed at the Institute for Microsystem Technology (IMTEK) and at Biofluidix in Freiburg, Germany. 